\title{
INFARCTION OF THE CARDIAC AURICLES (ATRIA): CLINICAL, PATHOLOGICAL, AND EXPERIMENTAL STUDIES
}

\author{
BY
}

\author{
E. H. CUSHING, H. S. FEIL, E. J. STANTON, AND W. B. WARTMAN
}

From the Departments of Medicine and Surgery and the Institute of Pathology of Western Reserve University and the University Hospitals of Cleveland, U.S.A.

Received December 8, 1941

Infarction of the auricles * (atria) of the heart is generally considered a rare lesion of little clinical importance and few cases are reported. However, a study of the records at the University Hospitals of Cleveland showed that the auricles were involved in 31 (17.0 per cent) of 182 cases of myocardial infarction that were proven at autopsy during the seven year period from 1934 to 1940 . This is the highest incidence of auricular infarction in any reported series, but is thought to be near the true incidence of the lesion, because during the period of investigation the auricles were specifically examined for infarcts.

In a previous publication (Feil, Cushing, and Hardesty, 1938) two cases of infarction of the right auricle were encountered in a pathological study of 34 cases of acute myocardial infarction. Bean (1938) reported a series of 300 cases of myocardial infarction in which two instances of auricular infarcts were encountered at autopsy. Auricular fibrillation was present in one of these cases. Clowe, Kellert, and Gorham (1934) described a verified case of rupture of the right auricle. They analysed 54 proved cases that had been reported and found that the rupture had occurred in the right auricle in 70 per cent of them. Laignal-Lavastine, Liber, and Bidou (1934) found a ruptured hæmorrhagic infarct in the right auricle, following thrombosis of the first portion of the right coronary artery and its auricular branches. Clerc and Levy (1925) studied a patient with mitral stenosis and infarction of the external and superior portions of the right auricle. The electrocardiogram showed what they termed " fibrillo-flutter." Lisa and Ring (1931) described rupture of the left auricle in a heart in which there was advanced coronary arteriosclerosis and a recent thrombus in the anterior descending branch of the left coronary artery. Daven-

* In the anatomical and pathological portions of this paper, starting with the section headed Pathological Observations on p. 20, a distinction is made between the words "atrium" and "auricle" in accordance with the B.N.A. terminology. Atrium is defined as the entire chamber, either left or right, at the base of the heart above the ventricle. The word "auricle" is reserved for the ear-like appendage of the atrium. In the discussion of the physiological and clinical aspects of the lesion, deference is made to long and common usage so that when the adjective "auricular" is employed it refers to the entire chamber and not to the appendage. Thus such terms as "auricular fibrillation," "auricular flutter" and "auricular mechanism" do not imply localization to the appendage. 
port (1928) reported 92 cases of proved cardiac rupture in which the right auricle was affected in 3 per cent and the left in 1 per cent. Krumbhaar and Crowell (1925) studied 22 cases of rupture of the heart and reviewed 632 reported cases. The right auricle was involved in 5 per cent and the left in 2 per cent, and infarction was mentioned as a frequent cause. Infarction of the auricular musculature was seen three times in von Glahn's (1936) pathological material.

Recently Langendorf (1939) studied a case of auricular infarction, which was verified by autopsy, and reported deviations from the isoelectric line of the $\mathrm{P}-\mathrm{Q}$ segment in the second and third leads of the electrocardiogram. In experimental injury of the atria, changes have been described in the $P$ wave and in the P-Q level by Hahn and Langendorf (1939). Abramson, Fenichel, and Strookhoff (1938) reported elevation of the P-Q interval (lead I) in dogs, following necrosis of the left atrium experimentally produced, and depression of the interval after necrosis developed in the right atrium. They suggested that the deviation of the $\mathrm{P}-\mathrm{Q}$ interval in man is due to necrosis of the auricle and is analogous to the deviation of the $\mathrm{S}-\mathrm{T}$ interval which occurs in ventricular ischaemia. In experiments in which the atrial arteries of rabbits were clamped, Lambert (1937) found changes in the auricular complex of the electrocardiogram. The most interesting phenomena were the depression of the $P-Q$ segment, which he termed "cupule," and occasionally a diphasic or inverted P wave. Conderelli (1929), on the other hand, was unable to produce electrocardiographic changes after temporary occlusion of the right atrial vessels. Sanders (1939) injected 95 per cent alcohol into the atria of dogs and found that nodal rhythm, wandering pacemaker, auricular extrasystoles, and paroxysmal auricular tachycardia developed when the necrosis involved the sinus node. Elevation of the auricular S-T segment in lead $I$ with an upward bowing and an auricular $Q$ wave frequently followed injection of the left auricle. Necrosis of the right auricle caused similar auricular S-T changes in leads II and III. Changes in the P wave consisted of broadening and inversion, diminution or increase in amplitude, and slurring and notching. Sometimes a $\mathrm{Q}$ or $\mathrm{S}$ wave was seen, or $\mathrm{M}$ or $\mathrm{W}$ complexes.

The present study was undertaken to determine: (1) the clinical and electrocardiographic criteria that are diagnostic of auricular (atrial) infarction; (2) the incidence of auricular involvement in autopsied cases of myocardial infarction; and (3) the electrocardiographic changes in experimental auricular (atrial) infarction.

\section{Clinical Observations in Human Auricular Infarction}

Thirty-one cases of auricular (atrial) infarction were found in 182 cases of myosardial infarction (17 per cent), culled from 2704 consecutive autopsies. Electrocardiograms were taken in 23 cases and the findings are given in Table I. Abnormal auricular mechanism was present in 17 of the 23 cases ( 74 per cent). On the other hand, in 91 verified cases of ventricular infarction without demunstrable auricular infarction, in which electrocardiograms had been taken, 

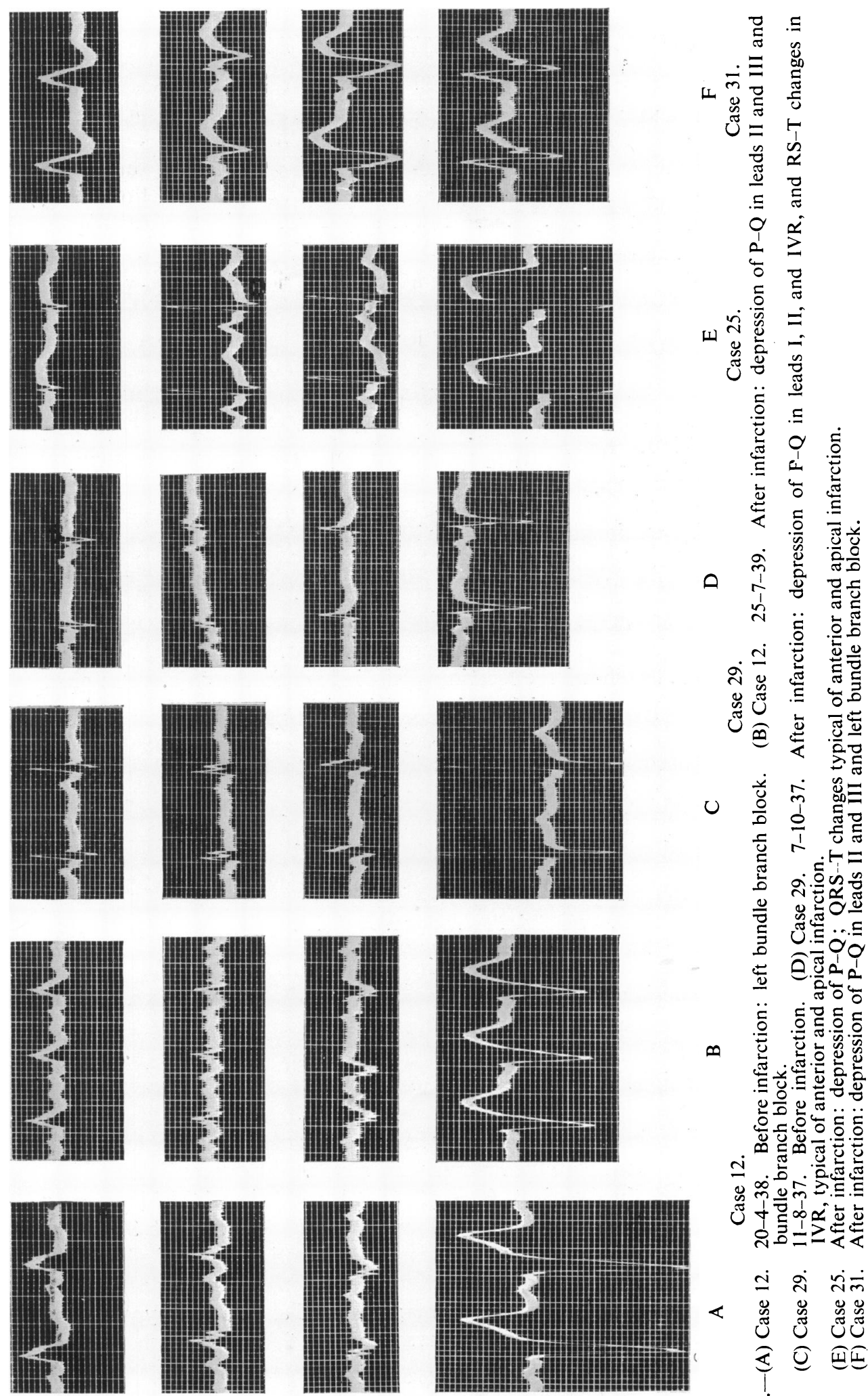

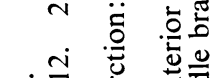

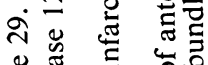

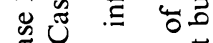

Uึ

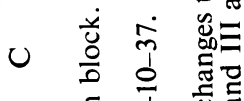

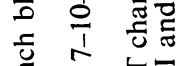

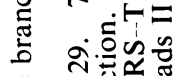
○ ํㅠㅁ

苛

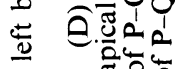

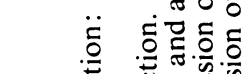

$\infty$ 这 吾诺. 응응 응

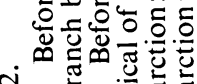

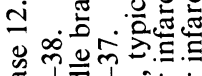

U +

ป तें

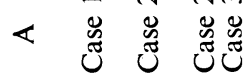

ङ 0 四吉

$\exists$

$\stackrel{n}{2}$

ن் 
there was evidence of disturbed auricular rhythm in only 8 cases ( 8 per cent). In the patients with infarcts of the auricles, auricular fibrillation was observed in 9 cases, auricular premature beats in 4, auricular flutter in 2, and sinus arrest and wandering pacemaker in 1 case each. The depression of the P-Q level (Fig. 1) described by Lambert and Langendorf occurred in 5 instances (22 per cent), but the value of this sign is doubtful because of its occurrence in normal people (Shipley and Hallaran, 1936). In the two cases with infarction of the sinus node, sinus arrest was present in one and auricular fibrillation in the other.

\section{Pathological Observations in Human Atrial Infarction}

A summary of the pathological observations in the cases of atrial * infarction is given in Table I. Before discussing these findings, it should be emphasized that many cases will be overlooked at autopsy, unless the existence of the lesion is kept constantly in mind. The gross recognition of atrial infarcts may be difficult and in questionable cases many blocks must be taken for microscopic examination. In this study, routine blocks were taken from the region of the sinus node, from both auricles, and from the lateral wall. Whenever mural thrombi were found in the atria, a suspicion of infarction was at once entertained and appropriate blocks were taken through such areas.

Fig. 2 (on p. 25) shows the location of the infarcts in the atria. The great majority of them were in the right atrium ( 27 times) in contrast to the occasional involvement of the left atrium (5 times). Most of the infarcts occurred in the auricles while the region of the sinus node was affected in only two instances. Multiple infarcts were found in two hearts.

Although the atrial arteries were carefully inspected after fixation by means of closely placed transections, gross occlusion was demonstrated in only one case. No doubt obstruction would have been found more frequently, had the coronary arteries been injected.

On the other hand, the anterior descending and circumflex branches of the left coronary artery, as well as the main right coronary artery were often diseased and serious stenosis or occlusion was encountered in 23 of the 31 cases. Either recent or remote infarcts of the ventricular myocardium were present in 22 of the cases. These were all in the left ventricle and interventricular septum, and often were multiple. Two other cases showed acute diffuse myocarditis and one chronic myocarditis. This left 6 hearts (Cases 3, 16, 17, 21, 26, and 28) in which auricular infarcts were the only lesions other than hypertrophy. With the exception of Case 26 all the infarcts were recent. Electrocardiograms had been taken in 4 of the 6 patients (Cases 3,16,21, and 26) and 2 had auricular fibrillation, 1 auricular flutter, and 1 low voltage of QRS. Case 3 died of heart failure; Case 16 suffered from malignant hypertension and died of uræmia; Case 17 had hypertension with cardiac decompensation, as well as acute pyelonephritis, and died of broncho-pneumonia; Cases 26 and 28 had hypertension and died of cardiac failure. It is, of course, unlikely that the auricular infarcts

* See footnote on p. 17. 
INFARCTION OF THE CARDIAC AURICLES (ATRIA) 21
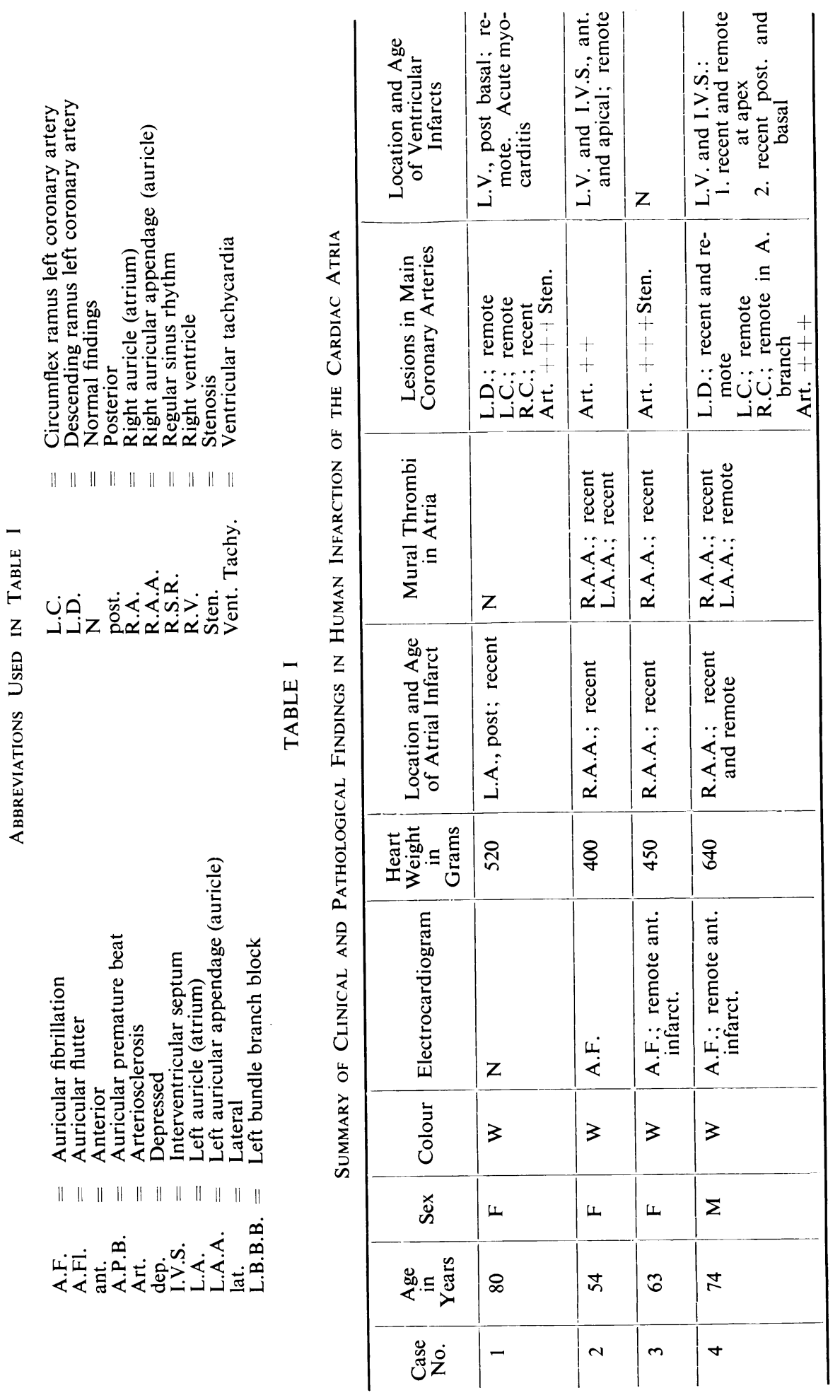

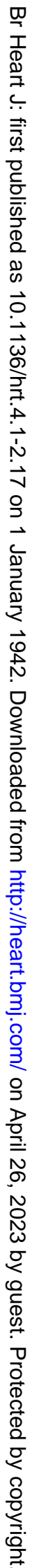




\begin{tabular}{|c|c|c|c|c|c|c|c|c|}
\hline 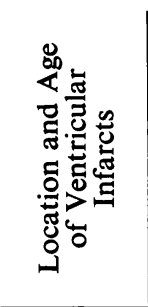 & 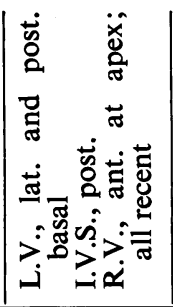 & 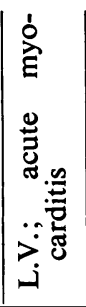 & 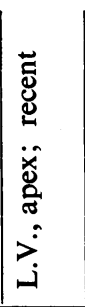 & 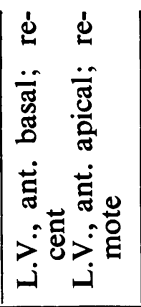 & 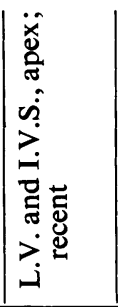 & 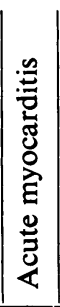 & 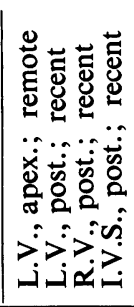 & 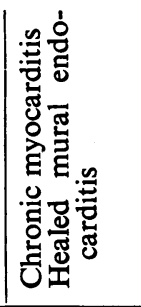 \\
\hline 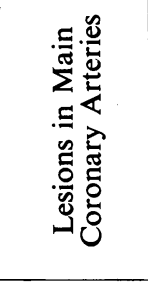 & 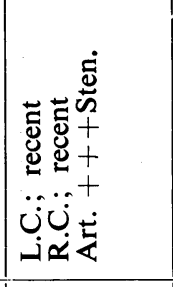 & $\begin{array}{l}+ \\
\dot{E} \\
\end{array}$ & $\begin{array}{l}+ \\
\dot{\vec{k}}\end{array}$ & 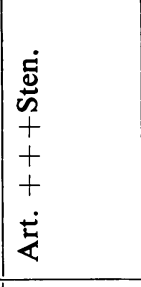 & 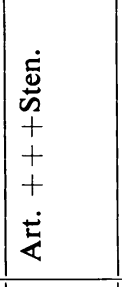 & $\begin{array}{l}+ \\
+ \\
\dot{z}\end{array}$ & 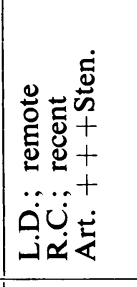 & $\begin{array}{l}+ \\
\dot{5} \\
\end{array}$ \\
\hline 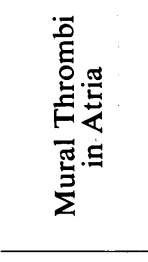 & 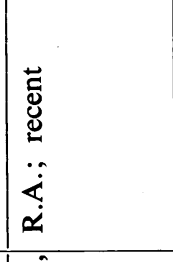 & 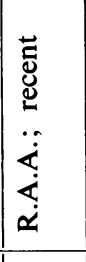 & 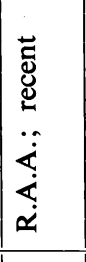 & 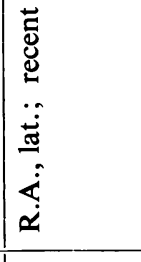 & Z & 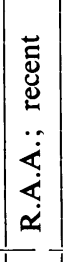 & $z$ & 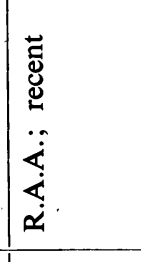 \\
\hline 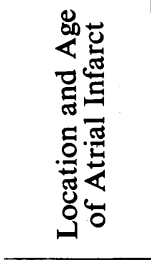 & 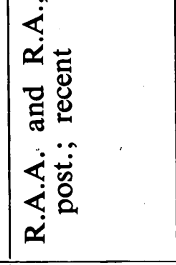 & 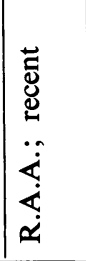 & 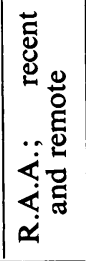 & 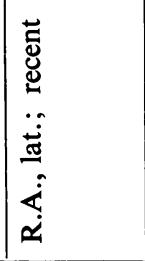 & 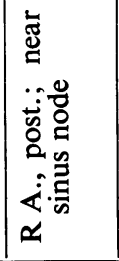 & 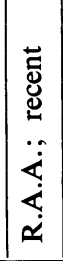 & 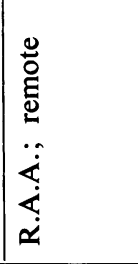 & 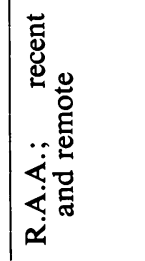 \\
\hline 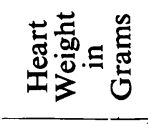 & 8 & 尽 & そ̊ & 总 & $\underset{\&}{\stackrel{\infty}{+}}$ & 号 & ৪্) & 8 \\
\hline 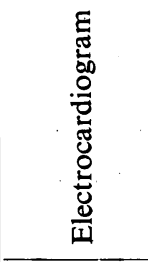 & 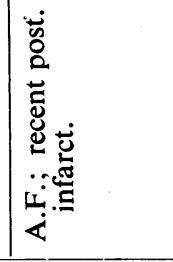 & $z$ & | & 送 & 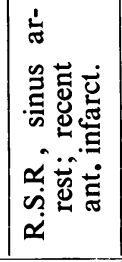 & $z$ & 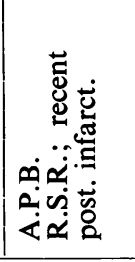 & 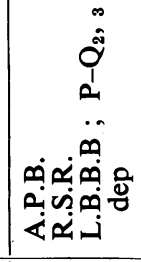 \\
\hline$\frac{0}{0}$ & 3 & 3 & $\infty$ & 3 & 3 & $\infty$ & 3 & 3 \\
\hline బ & $\Sigma$ & $\Sigma$ & $\Sigma$ & $\Sigma$ & L & $\Sigma$ & $\Sigma$ & $\Sigma$ \\
\hline 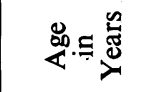 & 웅 & $\tilde{n}$ & $y$ & 范 & 8 & $\dot{\infty}$ & $\approx n$ & के \\
\hline 总 & in & 0 & $1-$ & $\infty$ & a & 은 & $=$ & $\simeq$ \\
\hline
\end{tabular}


INFARCTION OF THE CARDIAC AURICLES (ATRIA) 23

\begin{tabular}{|c|c|c|c|c|c|c|c|c|c|c|}
\hline 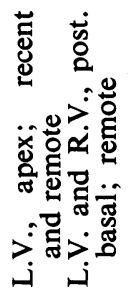 & 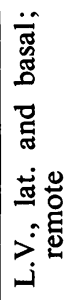 & 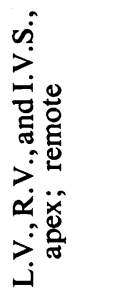 & $z$ & $\mathbf{z}$ & 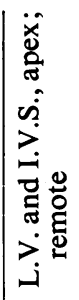 & 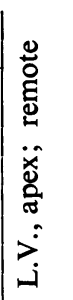 & 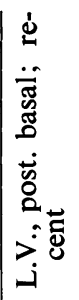 & $z$ & 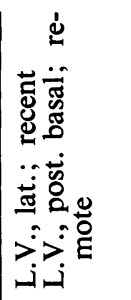 & 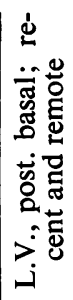 \\
\hline 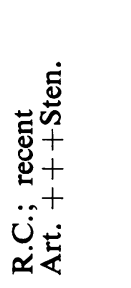 & $\begin{array}{l}+ \\
\dot{\vec{k}}\end{array}$ & 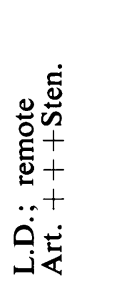 & $\begin{array}{l}+ \\
+ \\
+ \\
+ \\
\dot{z}\end{array}$ & $\begin{array}{l}+ \\
\dot{K}\end{array}$ & 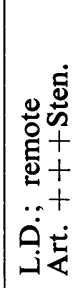 & 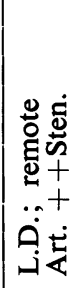 & 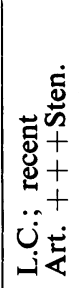 & 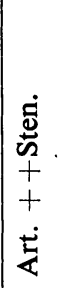 & 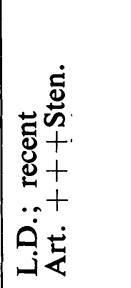 & 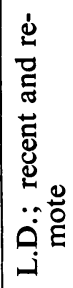 \\
\hline 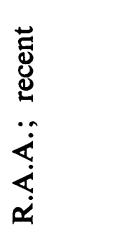 & 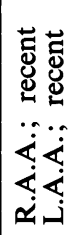 & $\mathbf{z}$ & 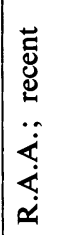 & 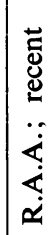 & 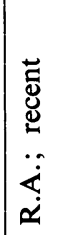 & $z$ & 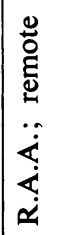 & $z$ & 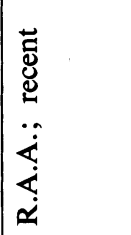 & $z$ \\
\hline 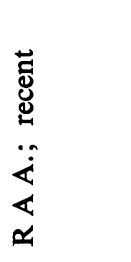 & 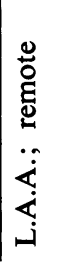 & 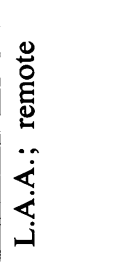 & 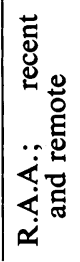 & 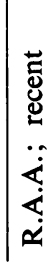 & 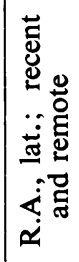 & 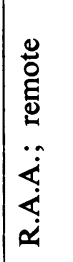 & 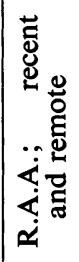 & 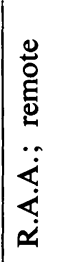 & 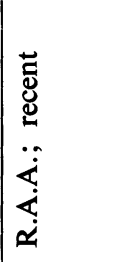 & 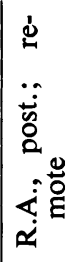 \\
\hline ষ্ণ & $\stackrel{\mathscr{D}}{\stackrel{2}{n}}$ & రి & 8 & \& & 영 & 总 & ஜ & \&్లి & ๙ి & 尽 \\
\hline $\mathbf{Z}$ & 芒 & 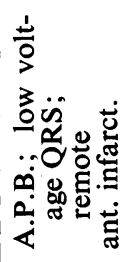 & $\begin{array}{l}\dot{x} \\
\dot{\alpha}\end{array}$ & $\mathbf{z}$ & 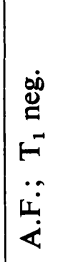 & $\mathbf{z}$ & $\begin{array}{l}\dot{0} \\
\tilde{\theta} \\
\ddot{0} \\
1 \\
0 \\
\ddot{\alpha} \\
\dot{\alpha} \\
\dot{a} \\
\ddot{\alpha}\end{array}$ & 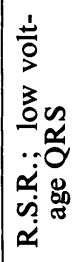 & 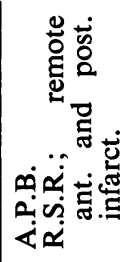 & $\mid \begin{array}{l}\dot{\alpha} \\
\dot{\alpha} \\
\ddot{\alpha}\end{array}$ \\
\hline 3 & 3 & 3 & $\infty$ & 3 & 3 & 3 & $m$ & $n$ & 3 & 3 \\
\hline$\Sigma$ & L & $\Sigma$ & L & $\Sigma$ & $\Sigma$ & $\Sigma$ & $\Sigma$ & L & $\Sigma$ & $\Sigma$ \\
\hline ర్ & $\stackrel{\infty}{n}$ & $n$ & 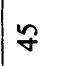 & $F$ & $g$ & 요 & $\infty$ & $\approx$ & $\stackrel{\infty}{\sim}$ & 2 \\
\hline$\underline{m}$ & 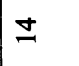 & $\simeq$ & 0 & $=$ & $\stackrel{\infty}{-}$ & 9 & శి & $\bar{\sim}$ & $\approx$ & $\approx$ \\
\hline
\end{tabular}




\begin{tabular}{|c|c|c|c|c|c|c|c|c|}
\hline 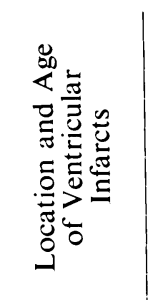 & 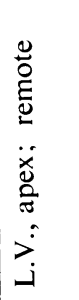 & 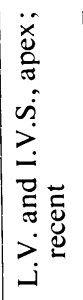 & $z$ & 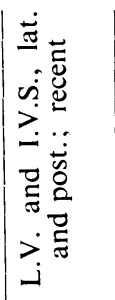 & $z$ & 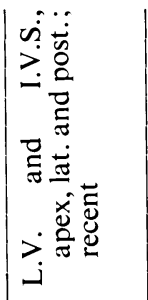 & 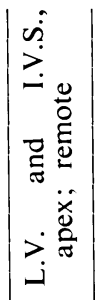 & 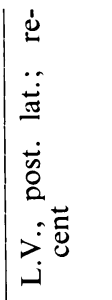 \\
\hline 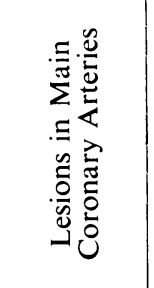 & 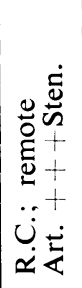 & 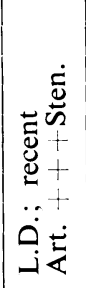 & 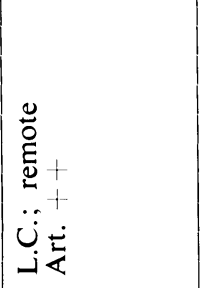 & 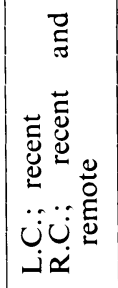 & $z$ & 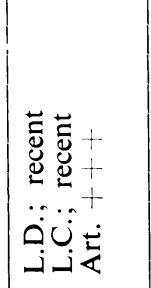 & 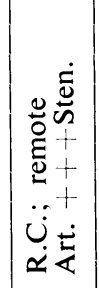 & 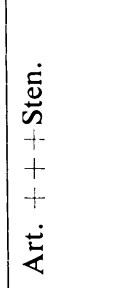 \\
\hline 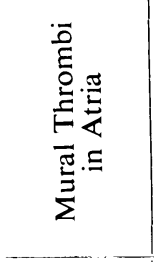 & 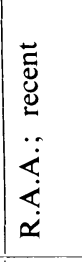 & 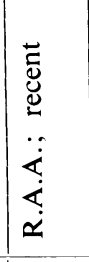 & 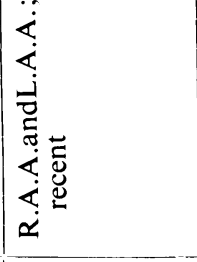 & 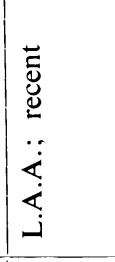 & 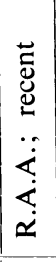 & 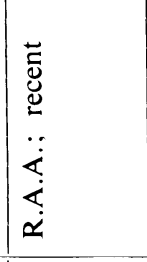 & 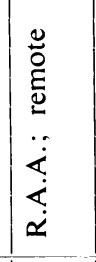 & 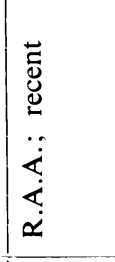 \\
\hline 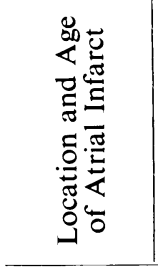 & 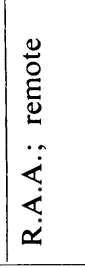 & 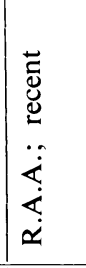 & 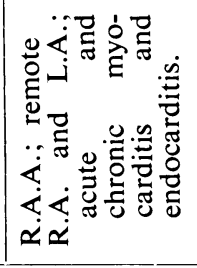 & 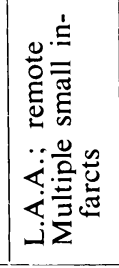 & 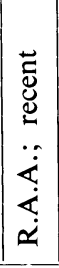 & 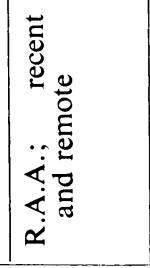 & 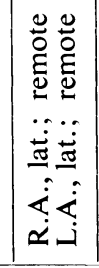 & 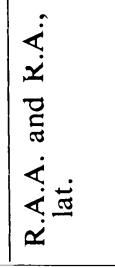 \\
\hline 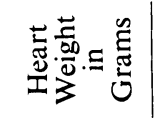 & ஜ̊م & $\underset{\sim}{\infty}$ & 8 & 点 & 8 & 号 & ஜ̊ & గ్గి \\
\hline 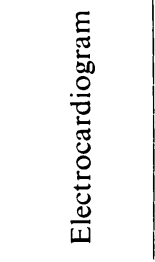 & $z$ & $\begin{array}{l}\dot{0} \\
\dot{\theta} \\
\ddot{0} \\
1 \\
\ddot{\vdots} \\
\ddot{\alpha} \\
\dot{\alpha} \\
\ddot{\alpha}\end{array}$ & 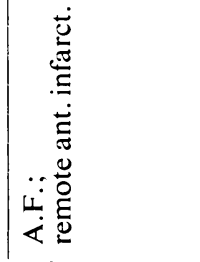 & 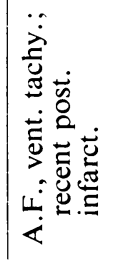 & $z$ & 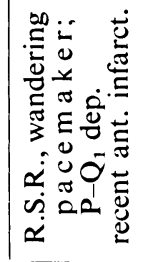 & $z$ & 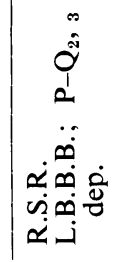 \\
\hline$\frac{\overline{0}}{0}$ & 3 & 3 & 3 & 3 & 3 & 3 & 3 & 3 \\
\hline $\begin{array}{l}\stackrel{x}{n} \\
\tilde{n}\end{array}$ & $\Sigma$ & $\Sigma$ & $\Sigma$ & $\Sigma$ & $\Sigma$ & $\Sigma$ & $\Sigma$ & $山$ \\
\hline 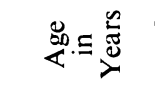 & $\pi$ & $i n$ & f & in & 8 & 요 & $n$ & 6 \\
\hline 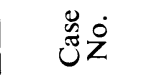 & $\underset{\sim}{ \pm}$ & $\approx$ & స్ర & $\bar{N}$ & $\stackrel{\infty}{\sim}$ & ते & ల & $\bar{m}$ \\
\hline
\end{tabular}




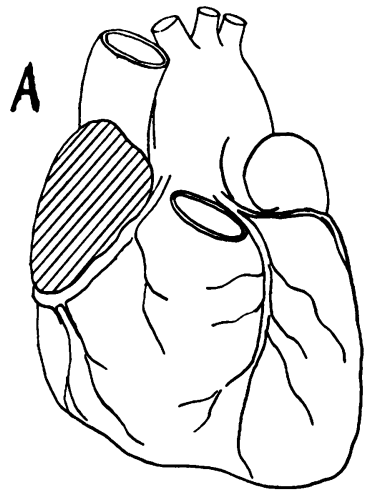

Right auricle.

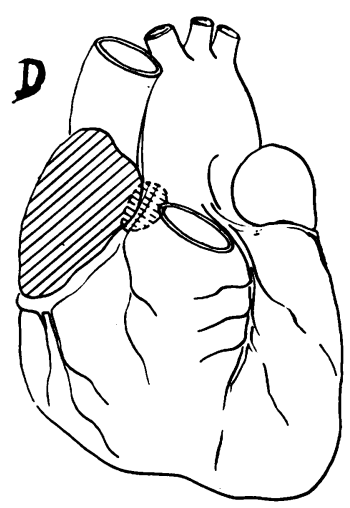

Multiple infarcts in right atrium. 2 ca:ses.

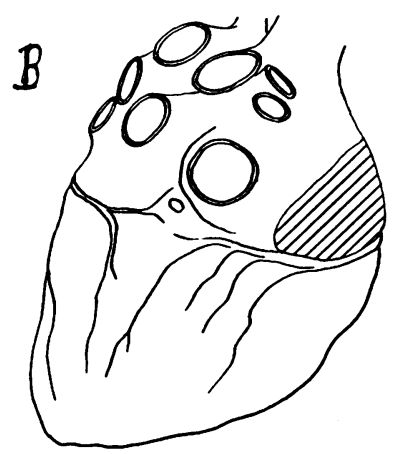

Rio̧ht atrium, lateral wall 2 coses.

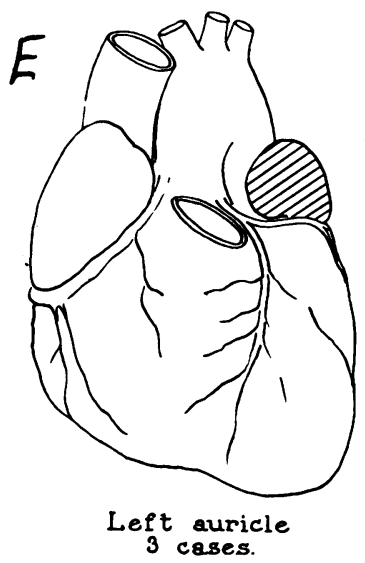

C

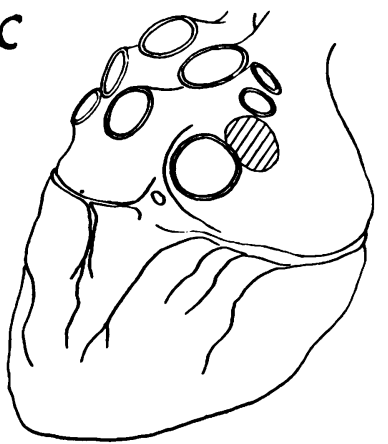

Right atrium, posterior wall involving sinus node.

2 cases.

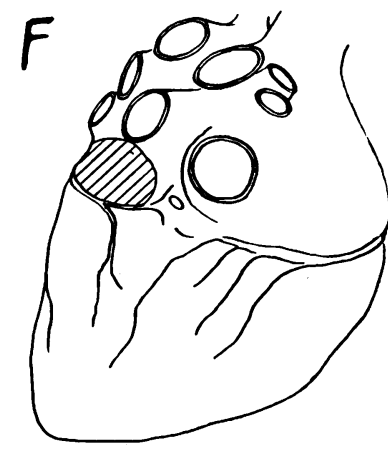

Left atrium, posterior wall 1 case.

FIG. 2.-Diagrams to show the situation of human atrial infarcts.

(A) Right auricle, 21 cases. (B) Right atrium, lateral wall, 2 cases. (C) Right atrium, posterior wall involving sinus node, 2 cases. (D) Multiple infarcts in right atrium, 2 cases. (E) Left auricle, 3 cases. (F) Left atrium, posterior wall, 1 case.

caused the death of these patients, but it is conceivable that they were a contributory factor.

The gross appearance of atrial infarcts was similar to that commonly seen in the ventricles (Fig. 3). It is worthy of note that in 26 cases, mural thrombi were found tightly adherent to the endocardium over the infarcted area.

Microscopically all stages of infarction were observed. The earliest changes consisted of hyperæmia and hæmorrhage with swelling of the muscle fibres and necrosis and exudation of leucocytes, followed later by hæmosiderin pigmentation and scarring. Often the muscle bundles in adjacent areas were swollen with vacuolated cytoplasm and large pleomorphic and hyperchromatic nuclei. In most cases the infarcts were massive, but occasionally small multiple lesions, separated from each other by intact myocardium, were observed. Massive infarction was usually accompanied by mural thrombosis of the endocardium, and when organization had occurred it was difficult to distinguish 

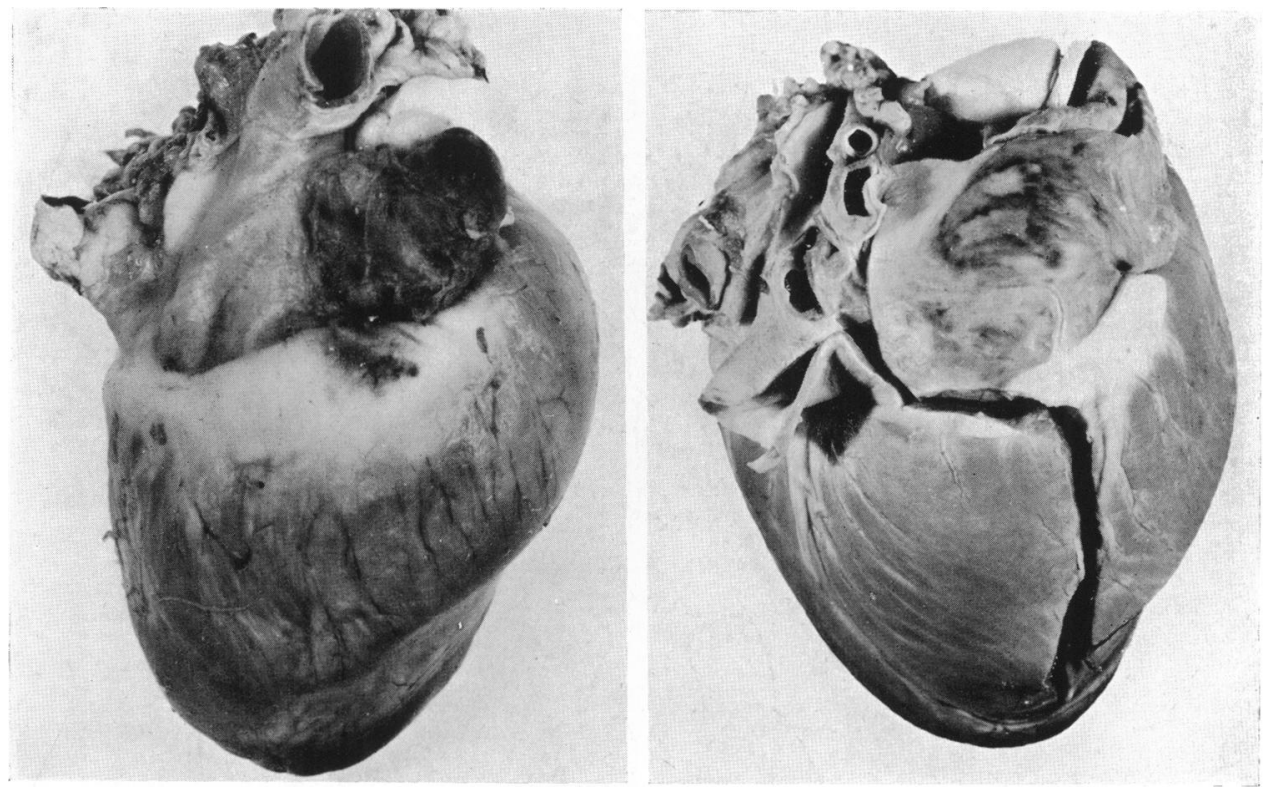

FIG. 3.-Atrial infarcts in dogs.

(A) Recent infarction in right auricle following experimental ligation of the main right coronary artery. A large infarct of the right ventricle was also present.

(B) Naturally occurring infarct in right auricle of a dog. No ventricular infarction. The localization of these lesions in the auricles, the dusky purple discoloration, and the presence of mural thrombi are also characteristic of the human lesions.

between necrotic myocardium and the thrombus. In such cases the Weigert stain was helpful, for by demonstrating the elastic lamina of the endocardium, the line of demarcation between muscle and thrombus could be clearly seen. When the infarct was not accompanied by mural thrombosis, there was nearly always a narrow border of surviving myocardium immediately beneath the endocardium. Microscopic study revealed small thrombotic arteries in several instances.

\section{Distribution of the Coronary Arteries of the Atria in Dogs AND IN MAN}

In this study of human atrial infarction the electrocardiographic changes described by Lambert and by Langendorf were not seen frequently. In view of this discrepancy, infarction of the atria of the heart was produced experimentally in dogs. Before describing the findings, it is necessary to review the distribution of the coronary arteries of the atria in dogs since it is different from that in man.

The atrial vessels of the dog's heart (Fig. 4) have a fairly constant distribution (Meek, Keenan, and Theisen, 1928-29). One or two vessels arise from the right coronary artery, shortly after its origin, and supply the surface of the right auricle and the superior vena cava. The lateral branch supplies the posterior 


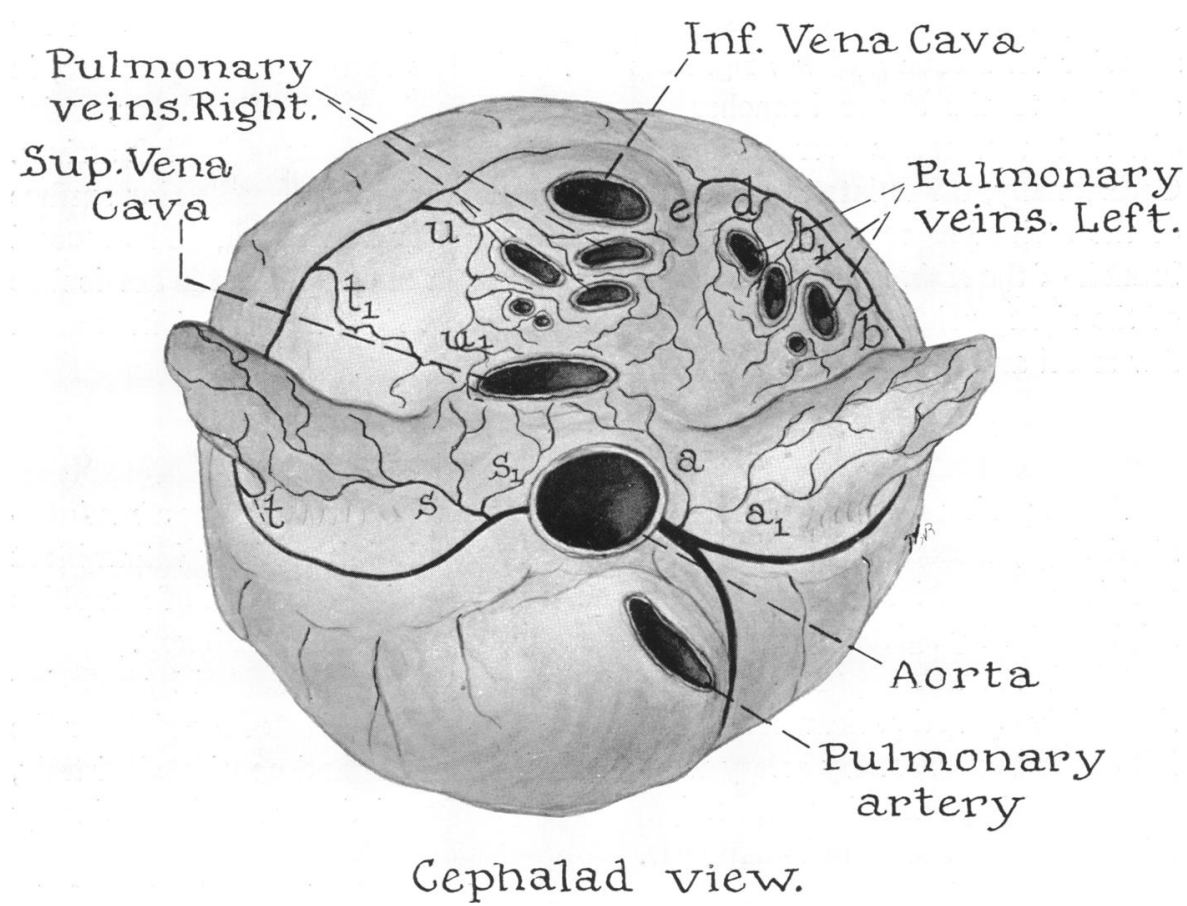

FIG. 4.-Distribution of the atrial branches of the coronary arteries in the dog.

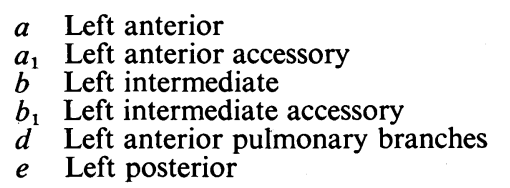

$s$ Right anterior

$s_{1}$ Right anterior accessory

$t$ Right intermediate

$t_{1}$ Right intermediate accessory

$u$ Right posterior

$u_{1}$ Sulcus artery

aspect of the right atrium, and there is a constant posterior branch that supplies the tissue about the pulmonary veins and anastomoses with the anterior branch about the superior vena cava. From the left coronary artery two branches arise close to the origin, one supplying the auricle and the other going upwards about the aorta to the vena cava. Posteriorly on the left side there is a lateral branch which ramifies about the pulmonary veins and goes upward toward the superior vena cava.

In man there are usually two branches from the right coronary artery (Gross, 1921). The first arises near its origin and comes behind the aorta, $5 \mathrm{~mm}$. above the auriculo-aortic groove. It supplies the anterior wall of the right atrium and sends branches to the auricle. It then passes through the septum to reach the posterior wall, where it surrounds in ring fashion the superior vena cava. This branch may arise from the left coronary artery near its origin; it encircles the base of the left auricle, ascending the external surface of the left atrium to the region of the superior vena cava, where it terminates as an arborization about the superior vena cava or passes immediately after its origin along the anterior wall of the left atrium to within $5 \mathrm{~mm}$. of the auriculo-aortic groove. On reaching the inter-atrial septum, it 
turns up and crosses on the superior aspect of the right atrium between the superior vena cava and the right auricle. The left coronary artery shows, in addition to the above branches, one or two smaller atrial branches, which distribute themselves over the anterior and superior surfaces of the left atrium. Occasionally, a left lateral atrial branch extends around the posterior surface of the atrium to reach the opening of the superior vena cava. The second branch of the right coronary artery is less constant and supplies the aortic face of the right auricle and the superior surface of the right atrium. There are a few small and inconstant atrial vessels from both sides.

\section{EXPERIMENTAL INFARCTION OF THE ATRIA}

All experiments were performed under aseptic conditions upon mediumsized dogs, anæsthetized with ether which was administered by intermittent positive pressure insufflation. Pre-operative medication consisted of morphine sulphate, $0.01 \mathrm{~g}$., given hypodermically one hour before the experiment.

The heart was exposed and supported in a cradle constructed by suturing the widely opened pericardium to the chest wall. Dissection of the right or the left circumflex coronary artery was begun at its origin and continued distally, each atrial branch being exposed, temporarily occluded several times, doubly ligated with fine silk, and finally cut.

Electrocardiograms were taken, employing the standard three leads and the chest lead IVR as follows: (1) a normal control before the start of the experiment; (2) one hour after the administration of morphine; (3) after ether anæsthesia; (4) after opening the chest; (5) after opening the pericardium ; (6) after construction of the pericardial cradle; (7) before and after each temporary occlusion of the individual atrial arteries; (8) before and after permanent ligation and section of each atrial artery; (9) at the conclusion of the experiment; and (10) daily thereafter. Chest leads were not taken after opening the thorax.

One or more atrial arteries were ligated in 18 dogs. Multiple operations at 12 to 21 day intervals on the same dog were performed and all right atrial branches were ligated in 11, all left atrial branches in 7 , and the arteries of both atria in 4 instances.

In four dogs the effect of chemically produced necrosis of the atrial myocardium was studied. In one animal various portions of the right atrium were painted with liquid phenol, and in three dogs the actual cautery was applied successively to the right and left atrium.

After sacrifice the hearts were injected through the right and left coronary arteries with barium sulphate gelatin after the method of Louis Gross (1921), chilled, X-rayed, and placed in 10 per cent formalin. Gross and microscopic examination of the hearts was made following the usual routine.

\section{Ligation of Right Atrial Arteries}

Dog 38-10. Artery $s_{1}$ ligated. Immediately afterwards $\mathbf{P}_{2}$ and $\mathbf{P}_{3}$ showed increased amplitude and returned to normal two hours later. There were no $\mathrm{P}-\mathrm{Q}$ changes. 
Daily electrocardiograms for twenty-two days showed no abnormality. Twenty-three days later, arteries $t$ and $t_{1}$ were ligated. After operation $P_{2}$ was slightly increased without $\mathrm{P}-\mathrm{Q}$ changes, and one day later the record was entirely normal. Twenty-one days later the animal was sacrificed and an organizing infarct of the right auricle and the atrium, as far as the acute margin of the heart, was found.

Dog 38-12. Arteries $s_{1}$ and $s$ ligated. The electrocardiogram showed slight elevation of $\mathrm{P}_{2}$ and $\mathrm{P}_{3}$ which persisted for twenty-four hours. Nineteen days later, pathological studies showed a small organizing infarct of the right auricle.

Dog 38-13. Artery $s_{1}$ ligated. No electrocardiographic change. Twenty-one days later, artery $t_{1}$ was ligated. The electrocardiogram remained normal.

Dog 38-14. Artery $s_{1}$ ligated. No electrocardiographic change.

Dog 39-273. Artery $t$ (small vessel) ligated. Wandering pacemaker developed and persisted about fifteen minutes. Following this, artery $u$ was ligated and the electrocardiogram showed slight changes in contour of $P_{1}$. Artery $t_{1}$ was ligated with no further change in the electrocardiogram. Artery $s$ was then ligated and auricular tachycardia developed. $\quad P_{2}$ and $P_{3}$ were broad and notched with increased amplitude. Forty-eight hours later the electrocardiogram was normal. After sacrifice the injected specimen showed that all right branches had been successfully ligated. Injection of the left coronary artery showed the barium to pass from the left atrial branches and fill the right atrial arteries. There was a recent infarct of the right auricle.

Dog 39-278. All right atrial arteries were ligated; following this slight but definite depression of $\mathbf{P}-\mathbf{Q}$ developed, and disappeared within twenty-four hours. Fifteen days later the dog was sacrificed, and injection showed that all arteries to the right atrium had been ligated. Excellent interatrial anastomoses with the left atrial artery were present. Microscopic studies showed infarction of the right auricle with beginning organization.

Dog 39-280. All right atrial arteries were ligated successively and nodal rhythm resulted. The dog died twelve hours after the experiment. Injection of the coronary arteries showed that all branches to the right atrium were successfully ligated. Microscopic sections were not obtained.

Dog 39-282. All right atrial arteries were ligated. The electrocardiogram remained normal throughout. Injection of the coronary arteries with barium showed all right atrial arteries to have been ligated. Microscopic studies showed a recent infarct of the right auricle.

\section{Ligation of Left Atrial Arteries}

Dog 39-258. Artery $a$ ligated and cut. No electrocardiographic change. The dog died of ventricular fibrillation during the experiment. Death was attributed to insufficient lung inflation.

Dog 39-260. Artery $a$ ligated. $\mathbf{P}_{1}$ became isoelectric, $\mathbf{P}_{2}$ and $\mathbf{P}_{3}$ were inverted, and the $P-Q$ interval remained normal. These changes persisted throughout the experiment. Artery $b_{1}$ was then ligated without further change. On the first day after operation, nodal rhythm appeared and persisted four days. From the fifth to the ninth days, wandering pacemaker was present. On the tenth day the rhythm became normal and remained so. At autopsy, recent infarction of the lateral wall of the left atrium was present.

Dog 39-259. Arteries $b_{1}$ and $b$ ligated. There were no electrocardiographic changes. Death occurred during the experiment as the result of pressure on the heart.

\section{Ligation of Right and Left Atrial Arteries}

The arteries of both atria were successfully ligated in four dogs. In one dog this was done in a one-stage operation and in three dogs by multiple stages. 
Dog 39-280. Ligation of arteries $u$ and $t$ produced no electrocardiographic changes. Following ligation of arteries $s$ and $s_{1}$ nodal rhythm resulted. Ligation of all left atrial arteries produced no further changes.

Dog 39-264. The first operation was performed on September 21, 1939, when artery $a$ was ligated, after which $\mathbf{P}_{1}, \mathbf{P}_{2}$, and $\mathbf{P}_{3}$ became isoelectric and triphasic with wandering pacemaker. Artery $b$ was then tied without significant change. After operation, wandering pacemaker and slight variation in size and shape of $\mathbf{P}_{1}, \mathbf{P}_{2}$, and $\mathbf{P}_{3}$ were present.

On October 2, artery $u$ was ligated without change in the electrocardiogram. A small artery proximal to $u$ was ligated and slight variation in contour of the $\mathrm{P}$ wave appeared. Ligation of artery $t$ produced no further change. After artery $s$ was ligated, $\mathbf{P}_{3}$ became diphasic with slight terminal depression. Ligation of artery $s_{1}$ produced no further change. After closure, 1:1 flutter developed (rate 279). On the first day after operation the rate was 220 with premature auricular beats. On the second and third days the rate was 226 and 284 respectively. On the fourth and fifth days A-V block, nodal extrasystoles, slight $\mathrm{P}-\mathrm{Q}$ depression, and prolonged $\mathrm{P}-\mathrm{R}$ interval $(0 \cdot 17)$ were present. On the following three days auricular flutter was present (auricular rate, 374; ventricular rate, 192). On the tenth day the rhythm was normal and remained so. At autopsy there were organizing infarcts in the lateral walls and auricles of both atria.

Dog 39-276. At the first operation on September 27, 1939, artery $s$ was ligated and the electrocardiogram remained normal. All remaining atrial arteries were then successively ligated without electrocardiographic change.

At the second operation on October 3 , arteries $a, b$, and $b_{1}$ were ligated. No electrocardiographic change occurred either during the experiment or after the operation. Twenty days later the animal was sacrificed and all the left and right atrial arteries were found to have been ligated. There were organizing infarcts in the right and left auricles.

Dog 39-267. The first operation was performed on September 25, 1939, and arteries $t$ and $u$ were ligated. The $\mathrm{P}-\mathrm{Q}_{2}$ showed a depression of $1 \mathrm{~mm}$., which continued for one day.

The second operation was performed on October 9 at which time arteries $a, b$, and $b_{1}$ were ligated. Only slight transient changes occurred in the contour of the $P$ wave, and these did not persist after the operation.

The third operation was performed on October 17 when arteries $s$ and $s_{1}$ were ligated. This was followed immediately by a low amplitude $\mathbf{P}$ wave which, however, returned to normal immediately after operation. On October 19 wandering pacemaker developed and disappeared within 24 hours, only to reappear on October 25.

Post-mortem organizing infarcts involving the lateral walls and auricles of both atria were found.

All the hearts were carefully examined for other evidence of disease, but none was found. No enlargement occurred. The main coronary arteries were widely patent and followed a normal distribution. No infarcts were found in either right or left ventricles or the interventricular septum and there was no myocarditis, endocarditis, or valvulitis. Numerous dense adhesions often containing foci of heterotopic bone were present in the atrio-ventricular sulcus at the operative site.

In all the experiments described so far, the main right and left coronary arteries were normal, which, of course, was very different from the human cases where the main coronary arteries were usually seriously diseased and showed marked stenosis. The human lesions were usually massive, were accompanied by mural thrombosis, and involved the endocardium. In the dogs, on 
the other hand, no mural thrombi were formed, the infarcts consisted of small multiple scars or foci of fresh necrosis separated by bands of healthy muscle, and there was always a narrow zone of healthy myocardium immediately beneath the endocardium. Because of these differences between the experiments in the dog and the spontaneous human lesions, ligation of the main right coronary artery close to its origin was carried out in three dogs.

Dog 41-290. Shortly after ligation, $S-T_{1}$ became depressed and this was followed by ventricular tachycardia. The rate was 422 per minute. This terminated in ventricular fibrillation and death, within a few minutes. The heart was not examined.

Dog 41-291. Three hours after operation, $P_{3}$ became higher and notched, and upper nodal rhythm developed. There was, in addition, slight depression of $R-T_{2}$ and $R-T_{3}$. Six days later upper nodal rhythm was noted. Thirteen days after operation regular sinus rhythm was present, and was noted again on the twenty-seventh day. On this later date the P-R interval was shortened $(0.07 \mathrm{sec}$.).

The animal was sacrificed on the thirtieth day and organizing infarcts were found in the right auricle and almost the entire right ventricle. The epicardium over these areas was thick, opaque, and pale grey, and there were numerous dense fibrous adhesions to the pericardium. The auricle was filled with a dark-red mural thrombus which on section showed well-developed organization.

Dog 41-305. Twenty-four hours after operation, upper nodal rhythm was observed with transient left bundle branch block. On the tenth day after operation sinoauricular block was present and four days later the cardiac mechanism was normal.

The animal was sacrificed on the fifteenth day. There was recent infarction of the right auricle, which was dark reddish-purple and covered with friable, yellowish-grey granular exudate. A moderately firm dark-red thrombus filled the auricle and was tightly adherent to the endocardium. Microscopic examination showed a recent hæmorrhagic infarct with mural thrombosis. A large recent infarct of the right ventricle was also present.

The infarcts produced in the two dogs which survived were identical with the human lesion. The auricle was involved in both cases. There was massive necrosis of myocardium and endocardium and there were organizing mural thrombi (Fig. 3).

\section{Heat and Chemical Necrosis of the Atria}

Heat cauterization of right and left atria. The inferior surface of the left auricle was cauterized and $P_{1}$ became diphasic and inverted. The superior surface of the left auricle was next cauterized and $P_{1}$ became isoelectric. After cauterizing the area below the left auricle and anterior to the pulmonary veins, $P_{1}$ remained isoelectric. The entire left atrium was then cauterized and ventricular tachycardia resulted. The right atrium was cauterized with no further changes and the experiment was terminated by ventricular fibrillation.

The lateral aspect of the right auricle was cauterized without causing any electrocardiographic changes. After cauterizing the medial aspect of the right auricle, no cardiographic change was noted. Following cauterization of the entire right auricle, auricular extrasystoles occurred. The body of the right atrium was burned and slight $\mathrm{P}-\mathrm{Q}$ depression resulted. At this point bleeding occurred from the necrotic wall, so that a clamp was placed across it, following which the electrocardiogram showed A-V nodal rhythm. After cauterization 
of the right atrium just above the vena cava the nodal rhythm persisted. The experiment was terminated by ventricular fibrillation and hæmorrhage from the atrium.

Phenol cauterization of right atrium. Liquified phenol was applied to the entire surface of the right atrium. The heart rate was decreased from 178 to 130 per minute. The mechanism changed from normal to upper nodal rhythm. $\mathrm{P}_{2}$ and $\mathrm{P}_{3}$ became diphasic, and the $\mathrm{P}-\mathrm{R}$ interval shortened from 0.09 to $0.06 \mathrm{sec}$. The dog died about one hour after conclusion of the experiment.

\section{Discussion}

A summary of the electrocardiographic changes following ligation of the atrial arteries and necrosis of the atria is found in Table II. No constant electrocardiographic patterns were noted, but the most frequent abnormalities were depression of $\mathrm{P}-\mathrm{Q}$, variations in the contour of $\mathrm{P}$, wandering pacemaker and $\mathrm{A}-\mathrm{V}$ nodal rhythm. As a control the electrocardiograms of 38 normal

TABLE II

Summary of Electrocardiographic Findings in Experimental Atrial Infarcts IN DoGS

\begin{tabular}{|c|c|c|}
\hline Electrocardiographic Alteration & $\begin{array}{l}\text { Frequency } \\
\text { of } \\
\text { Occurrence }\end{array}$ & Arteries Ligated \\
\hline $\begin{array}{l}\text { Transient increase in amplitude and } \\
\text { contour of } P \text { wave }\end{array}$ & 11 & $\begin{array}{l}s^{*}-9 \text { animals } \\
a^{*}-3 \text { animals }\end{array}$ \\
\hline Slight depression of $\mathrm{P}-\mathrm{Q}$ interval & 4 & $\begin{array}{l}s-1 \text { animal } \\
\text { All right }-1 \text { animal } \\
\text { Heat cautery of } \text { R.A. }-1 \text { animal }\end{array}$ \\
\hline Wandering pacemaker.. & 4 & $a-4$ animals \\
\hline Transient nodal rhythm & 3 & $\begin{array}{l}s-1 \text { animal } \\
a-1 \text { animal } \\
\text { Heat cautery R.A. }-1 \text { animal }\end{array}$ \\
\hline $\begin{array}{l}\text { Transient nodal rhythm and auricular } \\
\text { extrasystoles }\end{array}$ & 3 & $\begin{array}{l}\text { All right and all left }-1 \text { animal } \\
\text { All right }-1 \text { animal } \\
\text { Chemical necrosis of R.A. }-1 \text { animal }\end{array}$ \\
\hline Transient auricular tachycardia & 1 & $s-1$ animal \\
\hline Auricular flutter & 1 & All right -1 animal \\
\hline $\begin{array}{lll}\ldots & \ldots & \ldots\end{array}$ & 1 & All right -1 animal \\
\hline $\begin{array}{l}\text { A-V nodal rhythm and transient left } \\
\text { bundle branch block sino-auricular } \\
\text { block }\end{array}$ & 1 & $\begin{array}{l}\text { Right coronary artery ligated near } \\
\text { origin }\end{array}$ \\
\hline $\begin{array}{l}\mathbf{P}_{3} \text { notched } \\
\text { Wandering pacemaker } \\
\mathbf{R}-\mathbf{T}_{2}, \mathbf{R}-\mathbf{T}_{3} \text { slightly depressed } 6 \text { days; } \\
\text { after the operation upper nodal rhythm }\end{array}$ & 1 & $\begin{array}{l}\text { Right coronary artery ligated near } \\
\text { origin }\end{array}$ \\
\hline
\end{tabular}

* $s$, right anterior and $a$, left anterior, coronary arteries. 
dogs were studied. In nine dogs there was conspicious depression of $\mathrm{P}-\mathrm{Q}$, and there were a few instances of A-V nodal rhythm and wandering pacemaker.

The electrocardiographic findings in experimental injury of the atria were somewhat comparable to the changes in clinical infarction. It is interesting to note the absence of auricular fibrillation in experimental injury, although flutter and auricular tachycardia were each present once.

During the course of these experiments a case of naturally occurring infarction of the right auricle was observed in one of the animals in the dog colony of the Institute of Pathology (Fig. 3). This colony has been in existence for twelve years and has included one thousand animals. Eight hundred and fifty autopsies have been performed, and Dr. Harry Goldblatt, who has carefully examined the hearts, says he has never seen another case of spontaneous infarction of the atrium. This dog was being used in the course of other experiments in which blood had been injected into the media of the femoral arteries and thrombophlebitis had developed. Although the right coronary artery was carefully examined no obstruction was found. The infarct resembled the human lesions because of the presence of a large mural thrombus over the area of necrosis, because of its situation, and because there were no zones of intact myocardium.

The preponderance of infarction in the right auricle of man, which has been noted in this series as well as in reported cases, may be explained by the fact that when thrombosis occurs in the right coronary artery it is usually in the first two or three centimeters, thereby occluding the atrial branches that usually supply the auricle and $\mathrm{A}-\mathrm{V}$ node, although the left anterior atrial artery may also supply the latter area.

Curiously, while thrombosis of the left coronary artery and its branches occurred in 65 per cent of the cases in this series, the greatest incidence of atrial infarction was on the right side. This suggests that there may be other factors beside arterial thrombosis that will cause atrial infarction. Thus the nutrition of the atrial musculature, which may come in part through the Thebesian vessels or through the thin syncitium of the myocardium itself, may be interfered with by a large mural thrombus in the auricle. The high oxygen content of the arterial blood of the left atrium may prevent frequent infarction on this side.

We have been especially impressed with the fact that most of the cases of human atrial infarction reported in this paper have shown abnormalities of the auricular mechanism in the electrocardiograms. There have been auricular extrasystoles, auricular fibrillation, wandering pacemaker, nodal rhythm, and in five cases depression of the $\mathrm{P}-\mathrm{Q}$ interval.

\section{SUMMARY}

Infarction of the cardiac auricles (atria) was found in 17 per cent of 182 cases of myocardial infarction that were proven at autopsy. Abnormalities in the auricular complex of the electrocardiogram were present in 74 per cent of the cases of atrial infarction, but in only 9 per cent of all cases of 
infarction of the ventricles. Ligation of the atrial arteries in dogs produced abnormal auricular mechanism in only 6 out of 20 experiments. In 4 additional experiments there was a transient change in the contour of the $P$ wave. Depression of the $P-Q$ segment of the electrocardiogram was seen in 4 instances in which the atrial arteries were ligated. Abnormality in auricular mechanism is the most reliable clue to the clinical diagnosis of infarction of the atria.

\section{REFERENCES}

Abramson, D. I., Fenichel, N. M., and Shookhoff, C. (1938). Amer. Heart J., 15, 471.

Bean, W. B. (1938). Ann. intern. Med., 12, 71.

Clerc, A., and Levy, R. (1925). Bull. Mém. Soc. méd. Hôp. Paris, 49, 1603.

Clowe, G. M., Kellert, E., and Gorham, L. W. (1934). Amer. Heart J., 9, 324.

Condorelli, L. (1929). Z. ges. exper. Med., 68, 493.

Davenport, A. B. (1928). Amer. J. med. Sci., 176, 62.

Feil, H., Cushing, E. H., and Hardesty, J. T. (1938). Amer. Heart J., 15, 721.

von Glahn, W. C. (1936). Diseases of the Coronary Arteries and Cardiac Pain. The MacMillan Company, New York, p. 143.

Gross, L. (1921). The Blood Supply to the Heart. Paul B. Hoeber, New York.

Hahn, L., and Langendorf, R. (1939). Acta med. Scand., 100, 279.

Krumbhaar, E. B., and Crowell, C. (1925). Amer. J. med. Sci., 170, 828.

Laignel-Lavastine, Liber, A. F., and Bidou, S. (1934). Arch. Mal. Cour, 27, 580.

Lambert, J. (1937). Ibid., 30, 3.

Langendorf, R. (1939). Acta med. Scand., 100, 136.

Lisa, J. R., and Ring, A. (1931). J. Lab. clin. Med., 16, 1083.

Meek, W. J., Keenan, M., and Theisen, H. J. (1928-29). Amer. Heart J., 4, 591.

Sanders, A. (1939). Amer. J. med. Sci., 198, 690.

Shipley, R. A., and Hallaran, W. R. (1936). Amer. Heart J., 11, 325. 